\title{
HENRI BERR ET L'HISTOIRE DES SCIENCES
}

\author{
Michel BLAY
}

En 1947 paraissent, d'abord sous la direction de Pierre Brunet puis sous celle de René Taton et de Suzanne Delorme, les premiers fascicules de la Revue d'histoire des sciences. En soutenant la création de cette revue, Henri Berr et le Centre international de synthèse (CIS) ne font, après la guerre, que redonner vie à l'une des activités centrales du Centre, la promotion de l'histoire des sciences.

En effet, depuis la rédaction en 1898 de sa thèse latine consacrée à Gassendi ${ }^{1}$, Henri Berr a toujours attaché le plus grand intérêt à l'histoire des sciences et à la diffusion des travaux des historiens des sciences. Lors de la création, en $1900^{2}$, de la Revue de synthèse historique, Henri Berr ouvre largement les pages de sa revue à l'histoire des sciences. Il le souligne d'ailleurs dans l'introduction qu'il rédige dans le premier fascicule de la Revue d'histoire des sciences:

«Quand, il y a près d'un demi-siècle, j'ai cré la Revue de synthèse historique pour rapprocher, pour rassembler, dans une publication unifiante, toutes les spécialités historiques, pour qu'apparût, de façon éclatante, le vrai caractère de l'Histoire, pour que la Synthèse s'organisât, j'ai, naturellement, fait place à l'histoire des sciences ; j'ai même fait, tout de suite, à cette histoire, une place d'honneur ${ }^{3}$. "

1. Henri BERR, An jure inter scepticos Gassendus numeratus fuerit, Paris, Hachette, 1898; trad. fr. Bernard Rochot, Paris, A. Michel, 1960. Voir également « Gassendi, historien des sciences ", Actes du II Congrès de philosophie (Genève, 1905), section d'histoire des sciences, p. 855. La même sensibilité à l'histoire des sciences se trouve dans L'Avenir de la philosophie, Paris, Hachette, 1899 , livre II.

2. Le premier fascicule est daté de juillet-décembre 1900 . On peut également consulter Martin SIEGEL, «Henri Berr's Revue de synthèse historique», History and Theory, vol. IX, 1970, p. 322-334.

3. Revue d'histoire des sciences, 1947, p. 5. 
C'est par une série d'articles de Paul Tannery que l'histoire des sciences fait son entrée dans la Revue de synthèse historique; il s'agit principalement d'articles généraux présentant l'état des recherches historiques dans divers champs disciplinaires et visant à contribuer à l'élaboration de la «synthèse historique ». Dans son article « Mathématiques ${ }^{4} »$ Paul Tannery précise :

« J'ai tenté, dans ces quelques pages, d'exposer brièvement la situation actuelle de l'histoire de la mathématique, les desiderata qu'elle me paraît présenter, enfin de donner une idée des éléments qu'elle peut offrir à la synthèse historique, telle du moins que je la conçois. Je me suis, sous ce dernier rapport, à peu près borné cette fois à considérer la technique la plus élémentaire, celle du calcul ; je me propose de parler successivement, dans les revues suivantes, des diverses branches de la science dont l'histoire offre de même un intérêt général ou donne lieu à des conclusions d'une certaine importance ${ }^{5}$. »

Après ce premier article «Mathématiques », Paul Tannery donne, dans les fascicules suivants les articles : «Histoire de la géométrie ${ }^{6}$ », « Mécanique $^{7}$ », «Astronomie ${ }^{8}$ » et, finalement, «De l'histoire générale des sciences ${ }^{9}$ » qui devait être le texte de sa leçon inaugurale s'il avait été élu au Collège de France ${ }^{10}$. Ce dernier article - Paul Tannery meurt le 27 novembre 1904 - est un véritable manifeste en faveur de l'histoire des sciences et de son enseignement. Les premières lignes sont sans ambiguïté :

«Dans la vie de l'humanité, les sciences jouent désormais un tel rôle que leur histoire mérite évidemment d'être étudiée et enseignée au même titre que le sont, par exemple, l'histoire de l'art ou celle de la littérature. L'évolution d'un mode spécial de l'activité de l'esprit humain ne peut, en effet, être négligée vis-à-vis des autres, alors que ce mode a été, dès l'origine, un des facteurs essentiels du progrès vers la civilisation, et que l'avenir semble devoir lui ménager une prédominance de plus en plus marquée.

\footnotetext{
4. Paul TANNERY, "Mathématiques », Revue de synthèse historique, p. 179-195.

5. Ibid., p. 194.

6. Ibid., t. II, 1901, p. 283-299.

7. lbid., t. IV, 1902 , p. $191-204$.

8. lbid., t. VI, 1903, p. 301-316.

9. Ibid., t. VIII, 1904, p. 1-16. Déjà dans le t. VII (1903), à l'occasion de la publication d'un vœu relatif à l'enseignement de l'histoire des sciences émis par la Section d'histoire des sciences mathématiques, physiques, naturelles et médicales du Congrès des sciences historiques de Rome en 1903, Paul Tannery précise: «Le couronnement de cet enseignement, par la création de chaires d'histoires générales des sciences, est incontestablement le desideratum pour l'avenir, au point de vue où se place la Revue de synthèse historique, mais il est à coup sûr essentiel de commencer par organiser un enseignement relativement élémentaire, et nous ne pouvons dès lors que nous associer pleinement au vou du congrès de Rome», p. 101.

10. Cf. infra note 16.
} 
Je regarde comme inutile d'insister sur ce point. Mais en présence de ce fait que, jusqu'à présent, l'histoire des sciences n'a pas encore conquis, au milieu des autres histoires, la place qui lui est légitimement due, il convient d'en indiquer au moins les motifs, d'autant qu'ils doivent être pris en considération pour mieux orienter, si faire se peut, les travaux futurs ${ }^{11}$."

Quant au projet, présenté par Paul Tannery, d'une «Histoire générale des sciences », il restera au cœur des activités du CIS, comme en témoigne, par exemple, en 1935, la publication aux éditions Payot de l'Histoire des sciences. Antiquité, par Pierre Brunet et Aldo Mieli ${ }^{12}$. D'un certain point de vue, ce projet trouvera un aboutissement avec la publication, sous la direction de René Taton, à partir de 1957, des quatre volumes de l'Histoire générale des sciences ${ }^{13}$.

En 1906 paraît dans la Revue de synthèse historique un article signé par Abel Rey et intitulé «M. Duhem et l'histoire de la physique ${ }^{14} »$. Le rôle d'Abel Rey sera, après la guerre, considérable tant à la revue dans laquelle il publie en 1920 un important article «Revue d'histoire des sciences et d'histoire de la philosophie. Dans ses rapports avec les sciences ${ }^{15} »$, que dans les sections du CIS.

En rappelant d'entrée de jeu les noms de Paul Tannery ${ }^{16}$ et d'Abel Rey ainsi que l'importance que revêt l'histoire des sciences dans les fascicules

11. Revue de synthèse historique, t. VIII, 1904, p. 1.

12. Les divers comptes rendus de cet ouvrage figurent sous le titre «Pour une histoire générale des sciences ", Archeion, 1935, p. 266-274. Cette publication peut également être rapprochée, par son esprit, de celle de George SARTON, Introduction to the History of Science, 3 tomes en 5 vol., Baltimore, 1927-1948.

13. René TATON (éd.), Histoire générale des sciences, 4 vol., Paris, PUF, 1957-1964 (pour la 1 re éd.).

14. Revue de synthèse historique, t. XIII, 1906, p. 312-315. La même année, Abel REY a publié un «Pascal et l'expérience du Puy-de-Dôme », p. 143-160 qui provoque une réponse de Léon Brunschvicg, ibid., p. 363 sqq.

15. Ibid., t. XXX-XXXI, 1920, p. 121-135.

16. Henri Berr rend hommage à Paul Tannery à l'occasion de «l'inauguration solennelle du Comité international d'histoire des sciences » en 1929 qui coïncide avec une «commémoration » de Paul Tannery: «Quand j'ai fondé, il y a trente ans, la Revue de synthèse historique, destinée à embrasser toutes les manifestations de l'activité humaine et à rapprocher toutes les catégories d'historiens, Paul Tannery est un des premiers collaborateurs à qui j'ai fait appel ; et il m'a accordé son concours avec empressement parce que la synthèse historique, à laquelle il pouvait fournir un précieux apport, était sympathique à sa large intelligence. J'ai publié de lui, dans les tomes I, II, IV et V de la revue, une série de "revues générales" très remarquables, sur l'histoire des mathématiques, de la géométrie, de la mécanique, de l'astronomie. J'ai publié dans le tome VIII - j'ai tenu à publier, et j'en suis fier - la leçon inaugurale, sur l'Histoire générale des sciences, qu'il comptait faire, dans la chaire qu'il devait occuper au Collège de France, et qu'une injustice l'a empêché d'occuper.

II se plaignait dans cette leçon que 'l'histoire des sciences n'eut pas encore conquis, au milieu des autres histoires, la place qui lui est légitimement due". » Archeion, vol. XI, 1929, p. LXXVI. 
de la Revue de synthèse historique, je n'ai voulu que mettre rapidement en perspective les mots qu'Henri Berr prononce en 1929 à l'occasion de l'inauguration solennelle du Comité international d'histoire des sciences:

«M. Gino Loria ouvre la séance à $16 \mathrm{~h}$. Un nombreux public assiste à la séance. Une place d'honneur a été réservée à Mme Tannery.

Allocution de M. Henri Berr, directeur du Centre international de synthèse. Comme je l'ai fait déjà sans façon, officiellement, aujourd'hui, je souhaite la bienvenue dans cette maison aux membres du Comité international d'histoire des sciences. Je désire vivement, Messieurs, que vous vous y trouviez chez vous; je le désire en raison de la sympathie que nous éprouvons pour vos personnes et pour vos pays; je le désire en raison de l'importance que nous attachons à vos travaux. Ici même, avant-hier, j'ai essayé de montrer la place - qui est considérable - de l'histoire des sciences dans le système des connaissances, et, par suite, dans le programme de notre Centre de synthèse ${ }^{17}$.»

En soulignant tout l'intérêt qu'il porte à l'histoire des sciences et à ses chercheurs, Henri Berr montre ainsi clairement la nécessité qu'il y a à la fois de développer la recherche en histoire des sciences et de lui offrir des lieux institutionnels.

Dans la perspective de ce colloque et des préoccupations d'Henri Berr, deux questions s'imposent maintenant:

- Comment Henri Berr conçoit-il le rôle de l'histoire des sciences dans le cadre de ses réflexions d'historien et de philosophe?

- Comment a-t-il favorisé le développement et la diffusion de l'histoire des sciences?

\section{1. - L'HISTOIRE DES SCIENCES DANS LA PENSÉE D'HENRI BERR}

L'intérêt porté par Henri Berr à l'histoire des sciences apparaît tout d'abord par l'utilisation d'exemples, empruntés à l'histoire des sciences, lui permettant d'éclairer certains aspects de la méthode qu'il s'efforce de définir pour constituer son « histoire-science ${ }^{18} »$.

17. Archeion, vol. XI, 1929, p. LXXV.

18. «Il nous a semblé que, peu à peu, dans les discussions provoquées ou enregistrées par la revue, une doctrine se constituait, tout au moins que se formulaient des hypothèses régulatrices de la science. Nous avons même essayé de condenser les résultats de ce travail collectif, d'élaborer une logique de l'histoire-science », Revue de synthèse historique, $\mathrm{t}$. XXVII, 1913, p. 1. Voir également Henri BERR, L'Histoire traditionnelle et la synthèse historique, Paris, F. Alcan, 1921, p. 57 sqq. 
Ainsi dans La Synthèse en histoire, Henri Berr, pour préciser la spécificité de son approche vis-à-vis de celles du philosophe et de l'érudit, écrit :

«L'œuvre historique d'un philosophe ou d'un artiste est précaire et s'effrite sous la critique. La monographie d'un érudit, la plus humble dissertation établit des données à toujours. Et c'est là, sans doute, une satisfaction solide : posséder sur un point déterminé la certitude. Mais cette satisfaction, à bien y regarder, est vaine. Ces données qu'on établit, en soi, n'ont aucune valeur. C'est de la connaissance brute, ce sont les matériaux de la science : cela n'est point de la science.

Dans l'étude de la nature, cet axiome: Il n'y a de science que du général, est admis depuis longtemps. La constatation des faits empiriques prépare la théorie. On sait toute la distance qui sépare un Tycho Brahé d'un Copernic ou d'un Galilée. L'un a employé sa vie à observer les astres, à noter des positions, à inscrire des phénomènes; les autres ont mis à profit ses observations pour établir les lois de la mécanique céleste. Les botanistes et les zoologistes ont fait d'abord des descriptions et des nomenclatures; puis ils ont classé les espèces; et enfin sont venus des biologistes qui ont formulé des théories de la vie et de l'évolution. Jusqu'à preuve du contraire, il semble naturel d'admettre que l'érudition est simplement un travail préparatoire destiné à permettre dans ce domaine l'élaboration du général ${ }^{19}$.»

De même lorsque Henri Berr s'interroge sur le concept de loi en histoire il s'appuie sur l'histoire de la biologie et sur les récents travaux de Claude Bernard :

«Toutes les sciences ont commencé par être un amalgame semblable de conceptions a priori et d'inductions empiriques, de vérités partielles. Un corps de science se constitue définitivement par l'élimination des théories arbitraires et la coordination des résultats acquis. Prenons l'exemple de la biologie. En ce qui concerne la physiologie générale, le mérite insigne de Claude Bernard, c'est d'avoir contribué, plus que personne, à introduire le déterminisme scientifique dans une classe de phénomènes qui y paraissaient réfractaires, d'avoir ainsi délogé les spéculations hasardeuses; et c'est d'avoir organisé l'amas confus des connaissances positives, d'avoir institué l'étude générale de la vie, en articulant de façon judicieuse l'ensemble de ces phénomènes ramenés à l'unité. Structure, composition chimique, action du milieu sur l'irritabilité fondamentale, activité fonctionnelle : ces points de vue ont subsisté, depuis, dans l'étude de la vie, bien qu'elle ait été complétée, améliorée, et que, par exemple, la décomposition structurale ait été poussée au-delà de la cellule ${ }^{20}$. 》

19. La Synthèse en histoire. Son rapport avec la synthèse générale, Paris, A. Michel, 1953

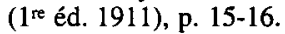

20. Ibid., p. 35. 
Cependant, au-delà de ce statut d'exemple, si l'histoire des sciences est importante pour Henri Berr, c'est, fondamentalement, dans le cadre de la «synthèse historique», parce qu'elle peut jouer un rôle central dans l'élaboration et la compréhension de l'histoire du progrès de la pensée. Il formule très clairement et fermement cette thèse dans En marge de l'histoire universelle:

«Nous croyons que, du point de vue qui est le nôtre - l'évolution de l'Humanité et, en particulier, le progrès du psychisme dans l'évolution humaine - l'histoire de la science n'est pas une chose morte, une curiosité d'érudit. Non seulement elle reproduit l'adaptation de l'esprit aux choses et la prise de possession par l'homme de son milieu, mais elle fait suivre la constitution d'une méthode destinée à résoudre les problèmes que se pose l'esprit, et qu'il prétendait trancher dans la religion d'abord, puis dans la métaphysique. La science amasse peu à peu des matériaux positifs qui permettent de soumettre à l'épreuve les vues $a$ priori de l'esprit : elle est bien un organum, l'instrument de résolution des problèmes philosophiques. L'histoire de la science est nécessaire à l'histoire de la pensée, parce que l'instrument du progrès de la pensée, c'est la science ${ }^{21}$.»

De même quelques pages plus loin :

«[...] nous reprendrons l'histoire des progrès de la connaissance et nous retrouverons le problème du rôle de la science. Il fallait marquer l'importance de l'histoire des sciences; il fallait l'assimiler dans l'histoire de la pensée humaine, dans l'histoire générale de la civilisation ${ }^{22}$.»

Plus précisément, encore, l'histoire des sciences est au cœur des recherches d'Henri Berr dans la mesure où, à travers celle-ci, il escompte saisir l'émergence et l'accomplissement de la pensée scientifique et logique. Ainsi, dans La Synthèse en histoire, après avoir commenté principalement les travaux de Lucien Lévy-Bruhl, Émile Durkheim et Marcel Mauss ${ }^{23}$, Henri Berr écrit:

21. En marge de l'histoire universelle, Paris, A. Michel, 1934, p. 232 (ce volume est constitué par les Avant-Propos donnés par Henri Berr dans les volumes de «L'Évolution de l'humanité »). Ce texte est, pour l'essentiel, repris p. 270. Sur la pensée d'Henri Berr ainsi que sur celle d'Abel Rey dans le contexte de la pensée de l'entre-deux-guerres, on peut consulter la très intéressante thèse d'Enrico Gastelli GatTinaRA, Crise de la raison et pensée de l'ouverture: Histoire et épistémologie en France dans l'entre-deux-guerres, thèse soutenue à Paris le 24 avril 1992.

22. En marge de l'histoire universelle, op. cit. supra n. 21, p. 235-236.

23. La Synthèse en histoire, op. cit. supra n. 19, p. 138 sqq. 
«La tendance qu'ont nos sociologues, pour justifier leur parti pris, à se cantonner dans les temps lointains et les sociétés inférieures n'est pas sans inconvénient - si le problème capital en histoire, à la fois au point de vue théorique et au point de vue pratique, consiste à chercher comment la logique s'épanouit en pensée et la pensée en science dans la civilisa$\operatorname{tion}^{24}$.»

Puis, quelques paragraphes plus bas :

«Jamais la pensée n'a été totalement indépendante du milieu social — de même qu'elle n'a jamais été à l'abri des contingences de toutes sortes : et ces influences sont à déterminer. Mais ce qu'il importe aussi et surtout de préciser, c'est le travail interne de la pensée. Il faut suivre l'évolution qui s'accomplit dans ce milieu - différent du milieu social - que constituent les hommes en tant qu'êtres pensants ${ }^{25}$.»

\section{Ou bien encore :}

« Encore une fois, pour observer l'attitude scientifique, nous nous contenterons de dire : c'est une hypothèse - mais c'est une hypothèse qui s'impose, qui veut être vérifiée et précisée - qu'il y a, dans la pensée réfléchie, une logique interne des idées. Il est permis de concevoir et de soutenir que, au cours de l'évolution, le besoin logique inhérent à la vie, qui s'est nourri des représentations des choses, qui a pris conscience de sa fonction dans son fonctionnement même, et est devenu besoin de savoir, en vient à contrôler et rectifier, dans la science, les idées que forme l'esprit, par des méthodes qui donnent de plus en plus le pouvoir ou la prévision.

Nous n'avons pas ici à justifier notre hypothèse : ce serait quitter la théorie de la synthèse et faire de la synthèse effective. Ailleurs, nous avons, pour une période restreinte - et encore trop étendue - pour les derniers siècles de la pensée, essayé de discerner, dans la trame complexe des idées, et de suivre, le fil logique : ce n'était là, bien entendu, rien de définitif ni de présenté comme tel, mais plutôt une sorte de programme et un appel aux travailleurs. Nous avons essayé aussi d'établir le rôle respectif de la religion, de la philosophie et de la science; et de montrer comment la raison, se rapprochant toujours davantage de la réalité, au lieu de construire, par application de ses principes, des systèmes d'idées sur des connaissances imparfaites, fonde sur des connaissances précises et sûres un système de vérité ${ }^{26}$, "

24. Ibid., p. 203.

25. Ibid., p. 204.

26. lbid., p. 213-214. Cf. également p. 220-221. 
À la fin de ce texte, Henri Berr ajoute dans une note qu'en ce qui concerne spécialement «l'histoire des sciences, centre de l'histoire de la pensée ${ }^{27}$ », il importe de consulter la conclusion que donne Abel Rey dans son livre La Théorie de la physique chez les physiciens contemporains. On y lit en effet quelques lignes très suggestives:

"Seulement, le mouvement de réaction contre le rationalisme métaphysique et transcendant n'aura pas été stérile. La critique qu'on lui doit prépare une synthèse philosophique qui concilie en les absorbant dans un point de vue supérieur les thèses extrêmes. Oui, la pensée humaine a une histoire qui compte lorsqu'on veut l'apprécier ou l'analyser ; et cette histoire, c'est, depuis que la science existe, l'histoire des sciences qui en est le centre ${ }^{28}$. »

Cette référence d'Henri Berr au livre d'Abel Rey, marquant sur ce point la proximité de leurs pensées ${ }^{29}$, est prolongée par les importants commentaires rédigés par Henri Berr dans En marge de l'histoire universelle à l'occasion des publications d'Abel Rey sur les origines de la science grecque dans la collection de «L'Évolution de l'humanité ${ }^{30}$ »; le rôle de l'histoire des sciences dans l'élaboration de l'histoire de la pensée y est, là aussi, nettement affirmé :

«Ce qui fera l'intérêt exceptionnel de cette histoire et son rapport étroit avec l'objet propre de notre cuvre - lequel consiste à expliquer, et autant qu'il est possible $d u$ dedans, l'évolution humaine -, c'est le point de vue où s'est placé Abel Rey. Il a conçu sa tâche comme étude de la croissance de l'esprit humain, dans son activité essentielle, de la genèse de la raison. Je ne crois pas que pareil effort d'intériorisation ait été tenté jusqu'ici par les historiens spécialisés dans l'“archéologie des idées scientifiques" — comme Rey a dit quelque part. De cette archéologie, il se sert, lui, pour reconstituer le mouvement, le progrès de la pensée scientifique ${ }^{31}$. »

De même, un peu plus bas, en rappelant à la fois le sens de sa tâche et l'importance du CIS, Henri Berr écrit :

27. Ibid., p. 214 , n. 1.

28. Abel REY, La Théorie de la physique chez les physiciens contemporains, Paris, 1907, p. 399.

29. Les rapports professionnels entre les deux hommes se détériorèrent quelque peu après qu'Abel Rey prit en 1932 la direction de l'Institut d'histoire des sciences et des techniques fondé par l'Université de Paris (13, rue du Four) et qu'il mit en route la publication, à partir de 1934, de la revue Thalès.

30. La Science orientale avant les Grecs, Paris, La Renaissance du livre, 1930, et La Jeunesse de la science grecque, Paris, La Renaissance du livre, 1933.

31. En marge de l'histoire universelle, Paris, A. Michel, 1934, p. 271-272. 
«L'effort de Rey pour marquer dans la constitution des sciences le progrès de la Science, dans le progrès de la Science celui de la Pensée humaine et de la civilisation intellectuelle, est propre à confirmer l'hypothèse directrice de l'Évolution sur le rôle et la primauté de la logique. Il est propre, en même temps, à justifier la fondation récente d'un organisme destiné, et à promouvoir l'histoire des idées, et à intensifier le travail millénaire de la Pensée - d'un Centre de synthèse. "La Société des sciences, dit-il (la Science), a, au-dessus des personnalités de chacune d'elles, sa personnalité propre, et comme sa conscience." Elles tendent vers cette "harmonie générale" que les systèmes philosophiques prétendaient imposer à l'esprit. Rey fait apparaître ici et il suivra dans les volumes ultérieurs le "drame de la connaissance", l'action positive de la "foi": "Le culte de l'intelligence (qui comprend à la fois l'observation précise et la logique organisatrice) est un culte comme tous les autres. La raison est une émanation du mysticisme, peut-être son fruit, le plus parfait d'ailleurs et le plus achevé - d'autant plus achevé qu'il est absolument séparé. Le fruit mûr abandonne l'arbre ${ }^{32}$ ".»

Ou bien encore, dans le deuxième volume, publié en 1953, de En marge de l'histoire universelle:

«L'histoire de la science dans l'Antiquité, que nous a donnée Abel Rey, et à laquelle nous avons fait délibérément une place si large dans 'L'Évolution de l'humanité", dépasse de beaucoup en importance les faits eux-mêmes - dont l'intérêt est déjà très vif. Elle constitue - nous l'avons précédemment souligné - une profonde analyse du développement de l'esprit humain. C'est comme telle qu'elle méritait toute l'ampleur qu'elle a prise ici. Cette histoire de la genèse de l'intelligence, qui prolonge l'histoire de la vie, est la vraie histoire de l'humanité ${ }^{33}$. "

\section{Et, finalement :}

«Il [Rey] insiste, dans des pages admirables, sur les principes directeurs de son œuvre; et du même coup il confirme la validité des nôtres : il justifie, une fois de plus, la place que nous lui avions faite - et par lui, à la Science dans "L'Évolution de l'humanité".

En termes frappants il distingue, parmi les travailleurs scientifiques, les artisans et les maîtres, parmi les historiens, les éndits et les penseurs. La véritable histoire des sciences est, avant tout, "l'histoire de leur esprit philoso-

32. Ibid., p. 281.

33. Ibid., $2^{e}$ vol., Paris, A. Michel, 1953, p. 203-204. Voir dans ce même volume, p. 220 et 230. On peut également consulter les très intéressantes discussions qui ont eu lieu aux séances de la Section d'histoire des sciences du CIS en 1934 et 1935, in Archeion, 1935, p. 82-84 et 259-265. 
phique". Suivre les progrès dans la connaissance de l'univers, grâce au progrès de la technique scientifique, c'est-à-dire des méthodes - car c'est là un des sens du mot "technique" -, voilà qui est infiniment plus important que de constater le progrès des techniques, au sens d'applications de la science. En reconstituant la marche de la Pensée dans la science, on se place au cour de l'histoire de la civilisation. Aussi l'humanisme d'aujourd'hui doit-il être, selon lui, centré sur la science et son histoire : "C'est la voie par où l'on peut sortir de survivances désuètes, et d'une culture qui, par ce qu'elle garde de formel, est à l'antipode du véritable humanisme" 34 . "

Cette conception générale de l'histoire des sciences, qui, dépassant la simple recherche antiquaire, vise à offrir des matériaux pour une histoire des progrès de la pensée, est également présente dans l'œuvre d'Hélène Metzger dont le rôle au CIS, et plus spécialement, comme nous le verrons, à la Section d'histoire des sciences du Centre, sera déterminant à partir de 1928. En effet, pour Hélène Metzger, comme pour Henri Berr et Abel Rey, bien qu'elle ajoute à ces thèses quelques nuances épistémologiques, ce qui compte, par-delà le travail d'érudition aussi approfondi qu'il puisse être, c'est le sens qu'il faut donner à ce travail, c'est-à-dire tout d'abord saisir dans leur genèse même les concepts et les théories, et puis, plus fondamentalement mais aussi corrélativement, éclairer les problèmes philosophiques centrés sur la « théorie de la connaissance scientifique » et sur « la marche de l'esprit humain ${ }^{35} »$.

«L'histoire des sciences doit-elle se résoudre à être un amas érudit et sans valeur de faits quelconques et inattendus qui amuseraient un vieux savant le dimanche après-midi? ou bien doit-elle se faire le soldat d'une théorie philosophique concernant le monde physique, la structure de l'esprit humain ou l'organisation sociale, abandonnant par cette attitude dévouée et servile sa personnalité propre et son effort autonome?

Entre les deux branches du dilemme [...], nous allons tenter de découvrir la route royale ou le sentier rude qui permettra à l'histoire des sciences de montrer que si elle ne peut espérer remplacer la philosophie, si elle ne peut abandonner entièrement l'a priori sans devenir inconsistante et vague, elle peut du moins éclairer la méditation du philosophe construisant la théorie de la connaissance ${ }^{36}$.»

34. En marge de l'histoire universelle, op. cit. supra $\mathrm{n} .31,2^{\mathrm{e}}$ volume, p. 238. Voir également $\mathrm{H}$. BERR, La Montée de l'esprit. Bilan d'une vie et d'une cuvre, Paris, A. Michel, 1955, p. 102 sqq.

35. Hélène METZGER, «La méthode philosophique dans l'histoire des sciences », Archeion, 1937, p. 205. Pour plus de détails voir en particulier M. BLAY, «Léon Bloch et Hélène Metzger: la quête de la pensée newtonienne », Études sur Hélène Metzger, Gad Feudenthal (éd.), Leyde, E.J. Brill, 1990, p. 67-84.

36. «Tribunal de l'histoire et théorie de la connaissance scientifique », Archeion, 1935, p. 5. 
Dans le prolongement de cette conception du rôle de l'histoire des sciences, qui, en particulier avec Abel Rey, Henri Berr et Hélène Metzger, ouvre cette discipline sur des perspectives qui lui permettent de dépasser le pur travail d'érudition, Henri Berr a également favorisé avec l'aide d'Abel Rey et d'Hélène Metzger mais aussi d'Aldo Mieli, le développement institutionnel de l'histoire des sciences; et cela, tant par la création du CIS et de sa Section d'histoire des sciences qu'en soutenant activement la mise en place de nombreuses relations et instances internationales.

\section{2. - HENRI BERR ET LA DIFFUSION DE L'HISTOIRE DES SCIENCES}

Comme nous l'avons déjà indiqué, la Revue de synthèse historique a largement ouvert ses pages à l'histoire des sciences; de même le journal Science, créé en 1936 par Henri Berr, a joué dans la diffusion des sciences et de leur histoire un rôle non négligeable, comme le rappelle ce dernier dans La Montée de l'esprit:

«[...] avant la dernière guerre j'avais créé un journal, Science, qui, sans en être une émanation, profitait des ressources intellectuelles du Centre; un journal destiné non pas à vulgariser, mais à diffuser la science, qui, dans un feuilleton, l'“Encyclopédie périodique", devait en établir, pour toutes les disciplines, un bilan annuel, alors que les articles étaient animés de l'esprit de synthèse ${ }^{37}$."

Cependant, c'est avec la création de la Fondation «Pour la science» et du CIS au cours des années 1924-1926 ${ }^{38}$ que la promotion de l'histoire des. sciences prend une ampleur considérable. Après quelques années de mise 142.

37. La Montée de l'esprit. Bilan d'une vie èt d'une cuuve, Paris, A. Michel, 1955, p. 141-

38. Le décret du Conseil d'État concernant la «Fondation pour la Science " date du 26 novembre 1926. Pour plus de détails sur ces questions, voir en particulier G. GeMELLI, «Le Centre international de synthèse dans les années trente ", Études sur Hélène Metzger, Gad Feudenthal éd., Leyde, E.J. Brill, 1990, p. 237-243 et "Communauté intellectuelle et stratégies institutionnelles : Henri Berr et la fondation du Centre International de Synthèse », Revue de synthese, 1987, p. 225-259. Voir également le gros volume de la Revue de synthèse publié en janvier-juin 1950 regroupant à la fois des articles relatifs au «cinquantenaire de la revue », aux «vingt ans du Centre » et ceux de la Quinzième Semaine de synthèse dont le sujet est «La synthèse, Idée-force dans l'évolution de la pensée ». Par ailleurs les textes relatifs à la «Fondation pour la science», aux statuts ainsi qu'au fonctionnement du CIS sont donnés dans le fascicule de décembre 1925 de la Revue de synthèse, p. 5-16. 
au point, le CIS se compose en 1929 de quatre Sections. Leur description en est donnée dans le numéro d'octobre-décembre 1929 de la revue d'Aldo Mieli, Archeion, créée en 1919 :

«Les sections du Centre sont au nombre de quatre. La première, fondée avec le Centre même, est, nous l'avons dit, celle de synthèse historique dont M. Henri Berr est le directeur et M. Lucien Febvre, de l'Université de Strasbourg, le directeur adjoint. Cette section comprend trente membres français et trente membres étrangers. Ensuite par les soins de M. Abel Rey, on a commencé à constituer la Section des sciences de la nature. On avait envisagé d'abord de lui adjoindre une sous-section d'histoire des sciences; mais plus tard, en vue de donner à l'histoire des sciences toute l'importance qui lui revient, la Section d'histoire des sciences a été constituée en section indépendante. La section des sciences de la nature ne s'est complétée que dernièrement; elle comptera trente membres français et trente membres étrangers. Elle est divisée en sous-sections selon les différents groupes de science. M. Rey, qui va diriger, comme nous le verrons tout à l'heure, une nouvelle section, a cédé la direction de la section à M. Paul Langevin.

La nouvelle Section qui vient de se constituer sous la direction de M. Abel Rey est celle de Synthèse générale. Il a été décidé que, pour le moment, cette Section et celle d'histoire des sciences fonctionneraient chacune avec dix membres français et dix membres étrangers. En outre, toutes les Sections peuvent s'adjoindre des associés en nombre illimité. Parmi les membres de la Section de synthèse générale, plusieurs, à commencer par le directeur s'intéressent vivement à l'histoire des sciences. Je rappelle seulement MM. L. Brunschvicg, Lalande, E. Meyerson, Arnold Reymond, Félix Sartiaux, Jules Sageret, etc.

Enfin sous la direction de M. Aldo Mieli s'est constituée définitivement ces jours-ci la Section d'histoire des sciences dont les membres sont: membres français : MM. Raymond Beaupin, Gabriel Bertrand, Pierre Brunet, Marcel Fosseyeux, Lucien Hahn, M. Laignel-Lavastine, Francisque Marotte, P. Menetrier, Mme Hélène Metzger, M. Ernest Wickersheimer; membres étrangers : MM. Joseph Bidez, Florian Cajori, Gino Loria, Aldo Mieli, Julius Ruska, George Sarton, Henry E. Sigerist, Charles Singer, Karl Sudhoff, Quito Vet$\operatorname{ter}^{39}$.»

Cet intérêt manifeste pour l'histoire des sciences incarné par le rôle décisif que doit jouer la Section d'histoire des sciences est affirmé nettement par Henri Berr en 1928 dans les procès-verbaux du conseil d'administration du CIS :

39. Archeion, vol. XI, 1929, p. 438-439. 
«L'histoire des sciences [...] a été trop peu cultivée et ne l'a été que d'une façon discontinue et tâtonnante ou encore trop purement technique. Or elle se trouve établir une étroite liaison entre les sciences de la nature et celles de l'humanité [...] pour la synthèse des connaissances qui est notre objet propre, pour la synthèse de la spéculation et de l'action qui est la fin dernière de nos efforts [...] rien n'a plus d'importance que l'histoire des sciences ${ }^{40}$. »

La création du CIS et conjointement de la Section d'histoire des sciences offre alors un lieu pour le développement de l'histoire des sciences tant en France que sur le plan international. C'est la raison pour laquelle, après qu'Aldo Mieli en eut lancé l'idée au VI ${ }^{e}$ Congrès des Sciences historiques qui s'est tenu à Oslo du 14 au 18 août 1928, le CIS est directement associé à l'organisation du premier Congrès international d'histoire des sciences qui a eu lieu à Paris du 20 au 24 mai 192941. Les dates du Congrès coïncident avec celles de 1'inauguration officielle du CIS (mercredi 22 mai à $16 \mathrm{~h}$ ); elles marquent également les premiers travaux officiels, sous l'impulsion d'Aldo Mieli, du Comité international d'histoire des sciences qui deviendra l'Académie internationale d'histoire des sciences dont le siège est toujours au 12, rue Colbert ${ }^{42}$.

La profondeur des liens qui régissent le fonctionnement et les activités de ces diverses instances apparaît clairement dans une convocation publiée dans le volume XI (1929) de la revue Archeion:

\author{
« Première session \\ du Comité international d'histoire des sciences \\ et \\ premier Congrès international d'histoire des sciences \\ Paris 20-25 mai 1929
}

Les membres effectifs et les membres correspondants du Comité international d'histoire des sciences sont convoqués à Paris au CIS (Hôtel de Nevers, 12 rue Colbert, Paris $2^{e}$ ) du 20 au 25 mai 1929 pour la première session dudit Comité.

40. Texte cité par G. Gemelul, op. cit. supra n. 38, p. 239.

41. Il importe de signaler que ce premier Congrès a été précédé de trớs sessions internationales regroupant les historiens des sciences. Ces sessions constituant des sections du Congrès international d'histoire comparée (Paris, 1900, $5^{\mathrm{e}}$ section), du Congrès international des sciences historiques (Rome, 1903, $8^{\mathrm{e}}$ section), du Congrès international de philosophie (Genève, $1904,5^{e}$ section). D'un certain point de vue, on peut donc dire que le congrès de Paris est aussi la $4^{\mathrm{e}}$ session internationale d'histoire des sciences. À chacune des sessions, un vou a été formulé concernant l'enseignement de l'histoire des sciences!

42. Sur l'ensemble des questions relatives à la mise en place des différentes instances, nous renvoyons le lecteur à la revue Archeion, vol. IX, 1928, p. 497-512; ainsi qu'aux Archives internationales d'histoire des sciences, vol. 43, nº 130, juin 1993. 


\section{HENRI BERR ET LA CULTURE DU XX` SIÈCLE}

Tous les historiens des sciences sont invités à participer à ce premier Congrès international d'histoire des sciences qui aura lieu à Paris du 20 au 25 mai 1929 au CIS [...].

Les historiens des sciences réunis pour le Congrès sont invités à participer à la Semaine de synthèse organisée les mêmes jours par le CIS. Pendant cette semaine seront soumis à la discussion les thèmes suivants : La civilisation et les civilisations - l'évolution ${ }^{43}$. [...]"

Par la suite, la Section d'histoire des sciences, dont Aldo Mieli devient le directeur et Hélène Metzger la très active secrétaire, va organiser régulièrement des réunions dont les comptes rendus figurent dans les fascicules de la revue Archeion:

«L'ACTIVITÉ DE LA SECTION D'HISTOIRE DES SCIENCES EN 1930-1931

La Section d'histoire des sciences, entrée dans sa deuxième année d'existence, a poursuivi en 1930-1931 l'accomplissement de son programme :

$1^{\circ} \mathrm{La}$ bibliothèque d'histoire des sciences, contenant les ouvrages réunis par son directeur M. Mieli, est maintenant ouverte aux travailleurs le jeudi de 14 à 17 heures. Quelques personnes sont venues y poursuivre leurs recherches.

$2^{\circ}$ Le répertoire d'histoire des sciences a été l'objet de nos soins les plus assidus; il comprend actuellement les fiches signalétiques de 10000 ouvrages ou articles intéressant quelque point de son domaine; à ces fiches signalétiques, groupées par ordre de matière et par nom d'auteurs, ont été jointes, quand cela a été possible, des notes explicatives ou critiques facilitant le travail des chercheurs; dès maintenant il peut rendre, et a rendu effectivement, de grands services. Il sera complété ultérieurement.

$3^{\circ} \mathrm{La}$ section s'est réunie cinq fois dans l'année pour discuter un problème d'histoire des sciences, ou de l'organisation générale des études d'histoire des sciences.

Les sujets étudiés cette année furent : Remarques sur l'introduction des thêries newtoniennes en France au Xville siècle (M. Brunet). - Qui était Socrate? (M. Mieli). - Les origines de la science et les pythagoriciens (M. Marotte). - La notion de système dans l'histoire et la philosophie des sciences (M. Dingler, professeur à l'Université de Munich).

Le 13 mai 1931, après avoir constitué le Groupe français des historiens des sciences, MM. Rey, Laignel-Lavastine et Marotte ont fait un exposé sur le rôle de l'histoire des sciences dans les universités et les établissements d'enseignement secondaire en France. Cet enseignement est très insuffisant actuellement et, pour la bonne harmonie des études, il serait nécessaire de l'organiser et de lui donner plus d'importance.

43. Archeion, XI, 1929, p. 87-88. 
À l'issue de la réunion, il est décidé à l'unanimité que le vœu suivant sera envoyé au ministère de l'Instruction publique :

"Le groupe français d'historiens des sciences, considérant qu'il est particulièrement opportun que l'histoire des sciences obtienne dans l'enseignement la place que mérite son importance dans l'histoire générale, émet le vœu " $1^{\circ}$ que soit créé un Institut d'histoire des sciences auprès de certaines Universités;

" $2{ }^{\circ}$ que l'histoire des sciences fasse partie de la formation des professeurs de l'enseignement secondaire."

Hélène Metzger ${ }^{44}$.»

Le volume 12 de 1930 de la revue Archeion publie l'allocution d'Aldo Mieli présentée à la séance d'ouverture de la section. On y lit en particulier :

\author{
«Fondation "pour la science" \\ Centre international de synthèse \\ Section d'histoire des sciences \\ (Communications officielles)
}

Séance du mercredi 22 janvier 1930

Assistent à la séance Mme Metzger et MM. Beaupin, Berr, G. Bertrand, Bouvier, Brunet, Ducassé, Fosseyeux, Laignel-Lavastine, Lut-Falla, Marotte, Mieli, Rey, Tolédano, Wickersheimer.

M. Mieli ouvre la séance en prononçant l'allocution suivante:

Messieurs!

J'ai l'honneur et le plaisir d'ouvrir aujourd'hui les séances de la Section d'histoire des sciences du Centre international de synthèse. Dès sa fondation en 1925 (les premières circulaires en font témoignage), la direction du Centre international de synthèse a souligné l'importance que les études qui nous intéressent ont pour l'œuvre de synthèse qui est le but final de nos efforts. Ainsi la Section des sciences de la nature, qui se formait par les soins éclairés de M. Rey, fit tout de suite une large place aux études de ce genre. J'eus alors le plaisir, tout en demeurant encore à Rome, de recevoir de la part de M. Rey l'invitation de participer aux travaux de la section comme membre étranger, et de me considérer dès lors (c'était en 1926) comme un collaborateur à l'œuvre si importante qu'on accomplit. Plus tard, m'étant désormais fixé en France, on m'invita à diriger la sous-section qui, dans la Section des sciences de la nature, était plus spécialement réservée à l'histoire des sciences. Mais cette sous-section devait bientôt assumer une place encore plus grande dans notre maison. En effet, en complétant l'organisation du Centre, depuis son installation à l'Hôtel de Nevers,

44. Compte rendu, rédigé par Hélène Metzger, dans le t. I de 1931 de la Revue de synthèse, p. 192-193. Le premier vœu sera exaucé avec la création de l'Institut de la rue du Four (cf. supra n. 29). 
on la transforma en une section particulière et indépendante qui, dès lors, put avoir son groupe spécial de membres. Ceux-ci, du reste, comme ceux des autres sections, doivent se considérer comme membres du Centre tout court, et, s'il leur plaît, ils peuvent participer à toutes les séances et tous les travaux qui se déroulent ici, sous la direction attentive et clairvoyante de M. Henri Berr. C'est ainsi que, d'accord avec lui et avec $M$. Abel Rey, qui dirigera dorénavant la Section de synthèse générale, $\mathrm{j}$ 'ai arrêté les noms des dix premiers membres français de notre Section, et ceux des dix premiers membres étrangers. Ces membres sont : Mme Metzger et MM. Beaupin, Gabriel Bertrand, Brunet, Fosseyeux, Hahn, Laignel-Lavastine, Marotte, Ménétrier et Wickersheimer pour la France; MM. Bidez de Gand, Cajori de Berkeley, Cal., Loria de Genova, Ruska de Berlin, Sarton de Cambridge, Mass., Sigerist de Leipzig, Singer de London, Sudhoff de Leipzig, Vetter de Praha et enfin moi-même, pour l'étranger. Ce sont ces membres que j'ai convoqués aujourd'hui pour la première fois.

[...] La Direction du Centre a eu l'amabilité de me nommer directeur de notre section. Je vous propose que le secrétaire en soit un de nos membres les plus actifs, qui depuis de longs mois m'a savamment aidé, soit pour organiser notre section, soit pour les travaux du Comité international d'histoire des sciences : Mme Metzger ${ }^{45}$. [...]»

Il importe aussi de souligner, comme le note très justement en 1952 Suzanne Delorme dans le numéro de la revue Osiris dédié à Henri Berr, que c'est au cours de ces années d'avant-guerre que s'est constituée la très importante bibliothèque d'histoire des sciences du CIS :

«[...] L'histoire des sciences a toujours vivement intéressé Henri Berr : n'at-il pas accueilli sans hésitation Aldo Mieli, réfugié d'Italie, qui fit don au Centre d'une grande partie de sa bibliothèque, noyau de ce qui est devenu depuis, grâce aux dons de Mme Metzger et de Pierre Brunet, grâce aussi aux apports de l'Académie internationale d'histoire des sciences qui y a son siège, la Bibliothèque d'histoire des sciences la plus riche de Paris, en ce domaine ${ }^{46}$. »

À partir des fascicules de l'année 1930, la revue Archeion porte en couverture la mention «organe officiel du Comité international d'histoire des sciences et de la Section d'histoire des sciences du Centre international de synthèse ». Les pages de la revue Archeion sont, avec aussi celles de la revue Isis créée par George Sarton en $1912^{47}$, d'une certaine façon, les

45. Archeion, XII, 1930, p. 50-56.

46. Suzanne DELORME, «Henri Berr », Osiris, X, 1952, p. 7. La revue Osiris comme la revue lsis a été créée par George Sarton; cf. infra n. 47.

47. Sur les relations entre George Sarton et Henri Berr on peut consulter S. DeLORME « La naissance d'Isis. Premiers rapports entre Henri Berr et George Sarton », Actes du XIt Congrès international d'histoire des sciences, 24-31 aout 1965, Varsovie, Varsovie, 1967, p. 223-232. 
archives de l'histoire des sciences en tant que discipline s'organisant institutionnellement.

Avec la guerre, l'exil vers l'Argentine et la mort d'Aldo Mieli, la revue Archeion disparaît. Deux revues la remplaceront après la guerre, d'une part, les Archives internationales d'histoire des sciences, organe de l'Académie internationale d'histoire des sciences et, d'autre part, la Revue d'histoire des sciences qui apparaît comme l'organe de la section d'histoire des sciences du CIS ${ }^{48}$.

En favorisant largement le développement et la diffusion de l'histoire des sciences en France comme à l'étranger, mais aussi en inscrivant l'histoire des sciences dans le champ des questions essentielles liées à l'histoire de l'humanité et à l'avènement de la pensée scientifique, Henri Berr et le CIS ont profondément marqué l'histoire tant institutionnelle qu'intellectuelle et conceptuelle de l'histoire des sciences. Aussi, pour conclure, je souhaiterais simplement rappeler les quelques lignes rédigées très probablement par George Sarton qui figurent en tête du volume $\mathrm{X}$ de l'année 1952 de la revue Osiris, volume dédié à Henri Berr: «Il a bien souvent expliqué la polarité de la synthèse historique et de la synthèse scientifique et les historiens de la science lui expriment leur reconnaissance ${ }^{49}$."

48. Cf. en particulier l'article introductif rédigé par Henri BERR, « Antécédents de la nouvelle Revue d'histoire des sciences ", et publié dans le fascicule $n^{\circ} 1$ de 1947, p. 5-8.

49. Osiris, X, 1952, Dédicace. Je remercie Mme Suzanne Delorme pour les informations et les conseils qu'elle a bien voulu me donner. 